\title{
Stabilization of 2D NSHP Recursive Digital Filters with Guaranteed Stability Using PLSI Polynomials
}

\author{
K. R. Santhi, ${ }^{1}$ M. Ponnavaikko, ${ }^{2}$ and N. Gangatharan ${ }^{1}$ \\ ${ }^{1}$ Faculty of Engineering, Kigali Institute of Science and Technology (KIST), B.P. 3900, Kigali, Rwanda \\ ${ }^{2}$ Bharathidasan University, Trichy, Tamil Nadu 620024, India \\ Correspondence should be addressed to K. R. Santhi, santhikr@yahoo.com
}

Received 14 August 2008; Revised 8 January 2009; Accepted 28 January 2009

Recommended by Dimitrios Tzovaras

Two-dimensional digital filters have gained wide acceptance in recent years. For recursive filters, nonsymmetric half-plane versions (also known as semicausal) are more general than quarter-plane versions (also known as causal) in approximating arbitrary magnitude characteristics. The major problem in designing two-dimensional recursive filters is to guarantee their stability with the expected magnitude response. In general, it is very difficult to take stability constraints into account during the stage of approximation. This is the reason why it is useful to develop techniques, by which stability problem can be separated from the approximation problem. In this way, at the end of approximation process, if the filter becomes unstable, there is a need for stabilization procedures that produce a stable filter with similar magnitude response as that of the unstable filter. This paper, demonstrates a stabilization procedure for a two-dimensional nonsymmetric half-plane recursive filters based on planar least squares inverse (PLSI) polynomials. The paper's findings prove that, a new way of form-preserving transformation can be used to obtain stable PLSI polynomials. Therefore obtaining PLSI polynomial is computationally less involved with the proposed formpreserving transformation as compared to existing methods, and the stability of the resulting filters is guaranteed.

Copyright $\odot 2009$ K. R. Santhi et al. This is an open access article distributed under the Creative Commons Attribution License, which permits unrestricted use, distribution, and reproduction in any medium, provided the original work is properly cited.

\section{Introduction}

Nowadays, signals have become an integral part in daily life. They are used to bear or convey information. Signals operate in diverse fields such as, speech communication, data communication, image processing, and so forth. A two dimensional (2D) signal processing is one such area which has received wide attention in recent years. Indeed, the processing of medical pictures, satellite photographs, radar and sonar maps, seismic data mappings, gravity waves data, and magnetic recordings are such good examples, in which $2 \mathrm{D}$ signal processing is needed. In all these applications, the design of $2 \mathrm{D}$ filters plays a central role $[1,2]$. Recursive filtering has often been proved to be more efficient than nonrecursive filtering [3]. The advantages of recursive filter over nonrecursive filter lie on the basis that, almost every 2D frequency responses, can be better approximated, by a rational transfer function of recursive filter, than by a polynomial transfer function of nonrecursive filter. Further, it is experimentally observed that, spectra and ideal filters, derived from real data tend to be better approximated by recursive filters than by nonrecursive filters [4]. However, the fundamental problems in $2 \mathrm{D}$ recursive filter design are those of synthesis and stability [3]. For recursive filters, nonsymmetric half-plane (NSHP) versions are more general than quarter-plane $(\mathrm{QP})$ versions in approximating arbitrary magnitude characteristics. The term nonsymmetric half-plane filters is used to describe semicausal filters. It is demonstrated that $[5,6]$ that 2D NSHP recursive filters outperform 2D QP recursive filters in terms of approximating more general frequency response specification.

The design of both versions of 2D recursive digital filters NSHP and QP prove challenging due to the absence of Fundamental Theorem of Algebra in two dimensions [5]. That is, higher-order polynomials in two variables may not be, in general, factored into lower-order polynomials. Thus, many design techniques for $2 \mathrm{D}$ recursive filters exploit $1 \mathrm{D}$ design technique either by mapping $2 \mathrm{D}$ characteristics into $1 \mathrm{D}$, or by employing separable 1D filters. Another problem with the design of $2 \mathrm{D}$ recursive digital filters is to ensure their 
stability at the beginning stage of the design [5] since it is computationally tedious to take care of stability constraints during the approximation stage. So it would be advantageous to devise a technique by which the stability problem is detached from the approximation one, and it is here that stabilization methods come into play [7-9].

Two of the popular stabilization methods, which were extensions from 1D to 2D namely, the 2D Discrete Hilbert Transform (DHT) and the PLSI-have been left unsolved, and not much research contribution was reported on these in 1990's [10]. Nevertheless, in 1999, the problem facing the 2D DHT method was resolved by DameraVenkata et al. [8]. The PLSI method, which was originally extended from the 1D Least Squares Inverse (LSI) method encountered much criticism. It is well known that, the LSI polynomial of an unstable $1 \mathrm{D}$ polynomial, which is devoid of zeros on the unit circle, is always stable (i.e., minimum phase), and that it approximates the inverse of the original unstable polynomial in magnitude spectrum [11]. Because of these two properties, LSI technique can be effectively used in stabilizing $1 \mathrm{D}$ recursive filters. By extending the 1D LSI result, Shanks et al. [12] introduced a $2 \mathrm{D}$ stabilization procedure based on a conjecture that the PLSI polynomial is minimum phase. The validity of Shanks' conjecture was never proved; but was provisionally considered to be true because it appeared as a natural extension of a well-known property of the corresponding one-variable problem (LSI). In addition, it had been upheld by a large number of numerical experiments without a single failure.

Genin and Kamp reported in $[13,14]$ counterexamples to Shanks' conjecture, and disproved the validity of Shanks' conjecture. It is then brought to light that Shanks' conjecture is valid on limited grounds for lower-order PLSI polynomials and special polynomials [10].

This paper demonstrates the stabilization of 2D NSHP recursive filters based on PLSI polynomials, using a new way of form preserving transformation. There are few methods available in the literature to form PLSI polynomials and stabilizing NSHP filters using PLSI polynomials, but they are often complex $[5,10,15,16]$. This paper introduces a new way of form-preserving transformation that can be used to obtain PLSI polynomials which correspond to the given unstable 2D NSHP filter polynomial in a simple way. It is proved that, with the new way of form-preserving transformation, to get a PLSI polynomial is simple, computationally efficient and the resulting NSHP filters are guaranteed to be stable, whatever may be the order of the filter in consideration.

This paper is structured as follows. In Section 2, some preliminaries and concepts of recursive computability, recursive filters (QP and NSHP), and important definitions concerning recursive computability. Section 3, discusses the concept of LSI polynomials, and the general procedure to obtain LSI polynomials in one and two dimensions. Section 4 introduces a new way of form-preserving transformation and the procedure to obtain PLSI polynomials for NSHP filters-first, second, and degree $N$ in general. Based on the new way of form-preserving transformation, the paper proposes new theorems which will be useful to obtain the PLSI in a simple and efficient way. In Section 5, some numerical examples are given and demonstrate that, the PLSI polynomial obtained using the new way of formpreserving transformation always leads to stable filters. Section 6 deals with the Lagrange Multiplier Method of testing stability, while computational complexities are discussed in Section 7. Section 8 summarizes the complete work and concludes it.

\section{Some Important Preliminaries and Concepts}

This section, discusses some important preliminaries and concepts concerning recursive computability, two versions of recursive filters (QP and NSHP) and key definitions.

In all discussions throughout this paper, it is assumed that $z$-transform is defined with positive powers of $z[8,17]$. Under this assumption, Stability $\Leftrightarrow A(z) \neq 0$, for $|z| \leq 1$ (for 1D filter) and Stability $\Leftrightarrow A\left(z_{1}, z_{2}\right) \neq 0$, for $\left|z_{1}\right| \leq$ $1\left|z_{2}\right| \leq 1$ (for 2D filter). Here, $A(z)$ and $A\left(z_{1}, z_{2}\right)$ are the denominator polynomials of $1 \mathrm{D}$ and $2 \mathrm{D}$ filter transfer functions, respectively. In $1 \mathrm{D}$ filters, when the transfer function, $H(z)$ is expressed as a rational function of $z$, the numerator polynomial does not affect the system stability. In 2D filters, conversely, the presence of numerator polynomial could sometimes stabilize an otherwise unstable system, even when there are no common factors between the numerator and denominator polynomials. Since this situation occurs very rarely, the numerator polynomial of the 2D filter transfer function is assumed to be unity [18]. Therefore, the transfer function of $2 \mathrm{D}$ filter is $\left(z_{1}, z_{2}\right)=1 / A\left(z_{1}, z_{2}\right)$.

Recursive Computability and Recursive Filters. The difference equation with boundary conditions plays a particularly vital role in digital signal processing, since it is the only practical way to realize recursive filters. The difference equation can be used as a recursive procedure in computing the output [18]. Recursive computability is defined as follows.

Definition 1. A system is said to be recursive computable when there exists a path in computing every output point recursively, one point at a time.

Consider the following difference equation which shows the input-output relation of certain linear time invariant (LTI) system:

$$
\begin{aligned}
y(m, n)= & \sum \sum_{(k, l) \in R} a_{k l} x(m-k, n-l) \\
& -\sum \sum_{(k, l) \in R-(0,0)} b_{k l} y(m-k, n-l) .
\end{aligned}
$$

In this form (1), the difference equation represents an algorithm for computing the sample of $y$ as a function of $(m, n)$. The systems for which the output samples are to be computed in this manner are recursively computable. 
This implies that, if a system is to be recursive computable, the output mask must have wedge-support. Both QP and NSHP possess a wedge-support output mask and, hence, they are both recursively computable $[5,6]$. Now we have the following definition.

Definition 2. A 2D LTI system is said to be a wedge support system when its impulse response $h(m, n)$ is a wedge support sequence. If all nonzero values in a sequence lie in the region bounded by two lines, and the angle is less than $180^{\circ}$ then the sequence will be called wedge support sequence.

A 2D filter with an impulse response $h(m, n)$ is called quarter-plane if, $h(m, n)$ has support only in the quarterplane or its rotations. Likewise, a 2D NSHP filter has an impulse response, which is nonzero only on a particular NSHP region. Still, by restricting the numerator and denominator polynomials of the transfer function to a finite region of an NSHP, the class of NSHP recursive filters can be implemented in a recursive fashion. Any wedge support sequence can always be mapped to a first quadrant sequence by a linear mapping of variables without affecting its stability $[6]$.

Definition 3. A quarter-plane is a region of the form $\{m \geq$ $0, n \geq 0\}$ or their rotations. A quarter-plane recursive filter is called spatially causal if the region of support is in the first quadrant. The causal filter is also called ++ recursive filter. The other possible quarter-plane filters are,+--+ , and -recursive filters.

Figures 1(a) and 1(b) below shows the ++ quarter-plane and the recursion using ++ filter, respectively, [6].

Definition 4. A nonsymmetric half-plane is a region of the form $\{m \geq 0, n \geq 0\} \cup\{m>0, n<0\}$ or their rotations.

An NSHP filter will have an impulse response which is nonzero only in a particular NSHP. Based on the region of support $R$, there are eight possible classes of NSHP filters, $[6,19]$. However, all discussions in this paper are based only on $R+\oplus$ class filter, and it should be noted that, the results of this class of filter are also extended to other seven classes of NSHP filters. Figures 2(a) and 2(b)show the $+\oplus$ NSHP region and the $2 \mathrm{D}$ recursion using $\mathrm{a}+\oplus \operatorname{NSHP}$ Filter $[6,19]$.

This paper focuses on NSHP filters and stabilizing unstable NSHP filters using PLSI polynomials. 2D NSHP polynomial and its region of support are defined as follows.

Definition 5. A 2D NSHP polynomial of degree $N$ [17] is given by

$$
A\left(z_{1}, z_{2}\right)=\sum_{R+\oplus}^{N} \sum_{R+\oplus}^{N} a_{m n} z_{1}^{m} z_{2}^{n}
$$

where, the region of support, $R+\oplus=\{m \geq 0, n \geq 0\} \cup\{m>$ $0, n<0\}[6,10]$.
The transfer function of the NSHP filter is given by

$$
H\left(z_{1}, z_{2}\right)=\frac{B\left(z_{1}, z_{2}\right)}{A\left(z_{1}, z_{2}\right)}=\frac{\sum_{R+\oplus}^{N} \sum_{R+\oplus}^{N} b_{m n} z_{1}^{m} z_{2}^{n}}{\sum_{R+\oplus}^{N} \sum_{R+\oplus}^{N} a_{m n} z_{1}^{m} z_{2}^{n}} .
$$

In NSHP filters, the output $y(m, n)$ can be calculated recursively using the $2 \mathrm{D}$ difference equation given as follows:

$$
\begin{aligned}
y(m, n)= & \sum \sum_{R+\oplus} a_{k l} x(m-k, n-l) \\
& -\sum \sum_{R+\oplus-(0,0)} b_{k l} y(m-k, n-l) .
\end{aligned}
$$

With these preliminaries, the succeeding section demonstrates the basic concept of LSI polynomials and the procedures to obtain LSI polynomials in one and two dimensions.

\section{Basic Concept of Least Squares Inverse Polynomials (LSI) in 1D and 2D}

The basic concept of LSI technique and the general procedure to form LSI polynomials in one and two-dimensions are the core of this section. Indeed in 2D, LSI is commonly known as PLSI.

Let $T^{2}$ to be the distinguished boundary of the bicircle defined by $\left[\left(z_{1}, z_{2}\right) ;\left|z_{1}\right|=1,\left|z_{2}\right|=1\right]$ and $\bar{U}^{2}$ to be the closed region given by $\left[\left(z_{1}, z_{2}\right) ;\left|z_{1}\right| \leq 1,\left|z_{2}\right| \leq 1\right]$.

Definition 6. If $A(z)=\sum_{n=0}^{N} a_{n} z^{n}$ is a given 1D polynomial of degree $N$, then the 1D polynomial $A^{\prime}(z)=\sum_{m=0}^{M} a_{m}^{\prime} z^{m}$ of degree $M$, forms the LSI of $A(z)$, if

$$
A^{\prime}(z) \approx \frac{1}{A(z)} \quad \text { for }|z|=1
$$

In order to obtain the LSI, $A^{\prime}(z)$ let it be formed that

$$
C(z)=A(z) \times A^{\prime}(z) \approx 1,
$$

and then form an error function $E$ from $C(z)$, such that

$$
E=\left(1-c_{0}\right)^{2}+\sum_{j>0} c_{j}^{2}
$$

To evaluate the coefficients $a_{m}^{\prime}$ 's of the LSI polynomial $A^{\prime}(z)$, minimize the error function $E$ given in (7) with respect to each $a_{m}^{\prime}$ to have the following linear system of equations:

$$
\frac{\partial E}{\partial a_{m}^{\prime}}=0
$$




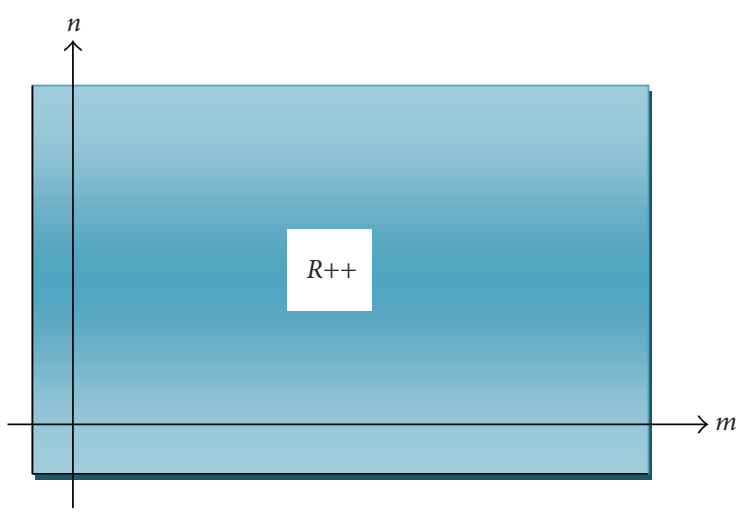

(a)

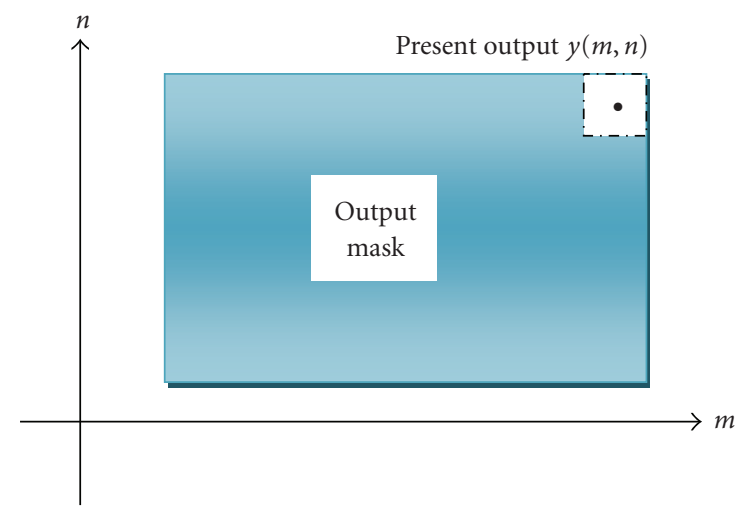

(b)

Figure 1: (a) The ++ Quarter-Plane. (b) 2D recursion Using ++ Filter.

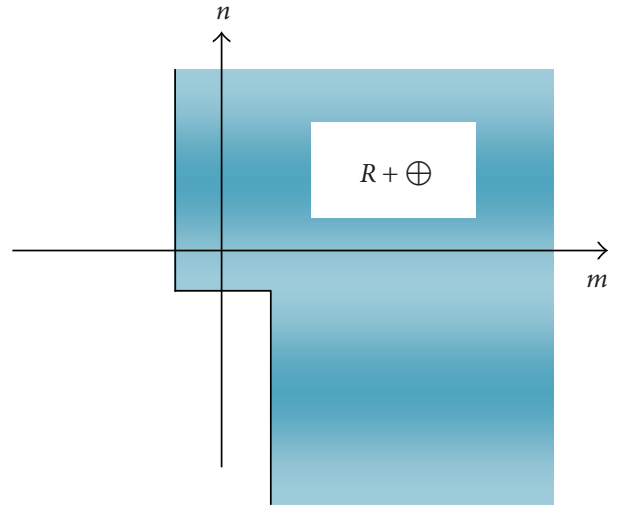

(a)

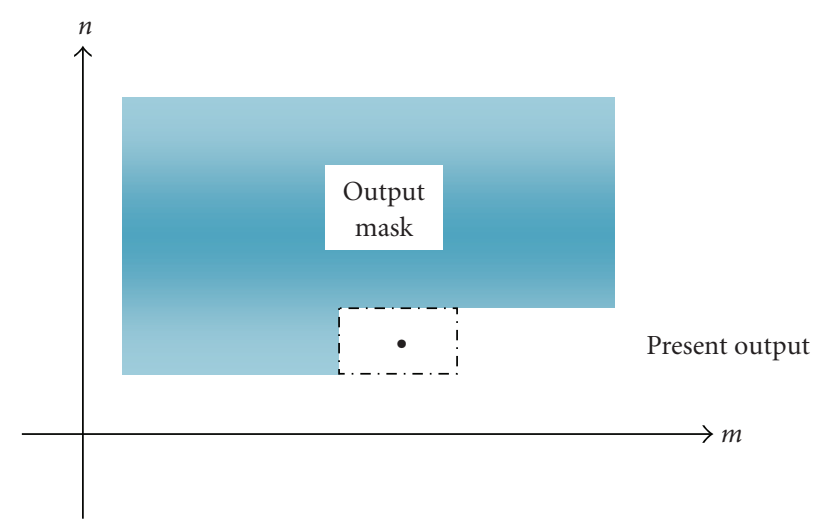

(b)

Figure 2: (a) The $+\oplus$ Nonsymmetric Half-Plane. (b) 2 D recursion using a $+\oplus$ Filter.

This will finally result in a set of linear algebraic equations (also known as normal equations) which is given in the form of matrix as follows:

$$
\bar{T} \overline{a^{\prime}}=\bar{a},
$$

where $\bar{T}$ is a square symmetric positive definite matrix (symmetric Toeplitz matrix) whose entries are functions of $a_{n}$ 's where $\overline{a^{\prime}}$ is a column vector of $a_{m}^{\prime}$ coefficients, and $\bar{a}$ is a column vector whose first entry is $a_{0}$ and the rest of $a_{n}$ 's are zeros. The solution of (9) will give all $a_{m}^{\prime}$ coefficients and, hence, the LSI polynomial, $A^{\prime}(z)$ which approximates $1 / A(z)$.

Expert literature demonstrates that, the 1D LSI polynomial, $A^{\prime}(z)$ corresponding to the unstable polynomial, $A(z)$ which is devoid of zeros on the unit circle is always stable $[11,20]$. This LSI method has been widely used to stabilize unstable $1 \mathrm{D}$ recursive filters.

The procedure to obtain the PLSI polynomial corresponding to a 2D NSHP polynomial is explored herewith.
Definition 7. If $A\left(z_{1}, z_{2}\right)=\sum_{R+\oplus}^{N} \sum_{R+\oplus}^{N} a_{m n} z_{1}^{m} z_{2}^{n}$ is a 2D NSHP polynomial of degree $N$ in both variables, then the $2 \mathrm{D}$ polynomial $A^{\prime}\left(z_{1}, z_{2}\right)=\sum_{R+\oplus}^{M} \sum_{R+\oplus}^{M} a_{m n}^{\prime} z_{1}^{m} z_{2}^{n}$ of degree $M$, forms the PLSI of $A\left(z_{1}, z_{2}\right)$ if

$$
A^{\prime}\left(z_{1}, z_{2}\right) \approx \frac{1}{A\left(z_{1}, z_{2}\right)} \text { for } T^{2}
$$

(see [10]). To obtain $A^{\prime}\left(z_{1}, z_{2}\right)$ just like in $1 \mathrm{D}$, first form

$$
\begin{aligned}
C\left(z_{1}, z_{2}\right) & =A\left(z_{1}, z_{2}\right) \times A^{\prime}\left(z_{1}, z_{2}\right) \approx 1, \\
C\left(z_{1}, z_{2}\right) & =\sum_{m=0}^{M+N} \sum_{n=0}^{M+N} c_{m n} z_{1}^{m} z_{2}^{n} \approx 1 .
\end{aligned}
$$

The approximation is achieved by choosing the coefficients of ' $\left(z_{1}, z_{2}\right)$, such that the error energy " $E$ " is minimized, 
where,

$$
E=\left(1-c_{00}\right)^{2}+\sum_{m=0}^{M+N} \sum_{n=0}^{M+N} c_{m n}
$$

Then differentiate $E$ with respect to each unknown coefficient $a_{m n}^{\prime}$ as follows:

$$
\frac{\partial E}{\partial a_{m n}^{\prime}}
$$

This will result in a set of linear algebraic equations of the form

$$
\bar{T} \overline{a^{\prime}}=\bar{a} .
$$

In (15), $\bar{T}$ is a square symmetric Toeplitz matrix whose entries are functions of $a_{m n}$ 's, $\overline{a^{\prime}}$ is a column vector of $a_{m n}^{\prime}$ coefficients as below:

$$
\overline{a^{\prime}}=\left\{a_{00}^{\prime}, a_{01}^{\prime}, a_{02}^{\prime}, \ldots, a_{m n}^{\prime}\right\}^{t},
$$

and $\bar{a}$ is also a column vector like

$$
\bar{a}=\left\{a_{00}, 0,0, \ldots, 0\right\}^{t}
$$

The solution of (15) will give all $a_{m n}^{\prime}$ coefficients and hence the PLSI, $A^{\prime}\left(z_{1}, z_{2}\right)$ which approximates $1 / A\left(z_{1}, z_{2}\right)$ [10].

In 1D, obtaining LSI polynomial is simple, but, it is demonstrated in [21] that obtaining PLSI directly from the 2D polynomial is extremely cumbersome, especially forming $\bar{T}$ matrix in the normal equation (15).

The concept of form-preserving transformation [20,21] is commonly employed to convert the $2 \mathrm{D}$ problem into $1 \mathrm{D}$ problem and therefore the stability of $2 \mathrm{D}$ is related to the stability of 1D LSI. Once it is converted to a 1D problem, the whole process of stabilization and stability testing is simple. This justifies the choice of going for form-preserving transformation. This paper compares the computations involved in obtaining the PLSI using the existing formpreserving transformation reported in [10] and the one used in this paper.

The following section, introduces the new way of formpreserving transformation that can be used to obtain the PLSI in a simple manner. Based on this transformation, some theorems have been proposed.

\section{Form-Preserving Transformation and Stability of PLSI Polynomials}

The literature on the concept of form-preserving transformation is used in different contexts for first quadrant and
NSHP recursive digital filters. For instance, Shanks [22] writes on techniques to design stable two-dimensional recursive filters. He demonstrates that, one-dimensional filters can be converted into two-dimensional filters with arbitrary directivity in a two-dimensional frequency response plane. In Shanks paper, and in subsequent ones [15, 23], the entire discussions focus on "one-quadrant" or quarter-plane recursive filters only. In [10], the concept of form-preserving transform was used in the stabilization of 2D NSHP recursive filters.

The PLSI method of stabilizing NSHP recursive filters reported in [10] has the following shortcomings:

(i) the obtained LSI is always suboptimal;

(ii) computational complexity is obvious.

In [10] to obtain the PLSI polynomial, the form-preserving transformation, $A^{\prime}\left(z^{L}, z\right)$ where $L=4 N+1$ is applied. This always leads to suboptimal (or constrained) polynomials and does not guarantee stability. A suboptimal polynomial is not always stable though it is reported in [10] as "always stable." This is a serious error. Unfortunately the authors of [10] have overlooked the fact that the 1D LSI polynomial $B(z)$ generated by the form preserving transformation is lacunary (missing some powers of $z$ or some powers of $z$ are forced to have zero coefficients). Hence the stability of such 1D LSI polynomials cannot be guaranteed though in some cases one may get stable polynomial in spite of the constraints. So the form preserving transformation reported in [10] is no longer applicable in the procedure for getting an always stable PLSI. More details about suboptimal and lacunary polynomials are reported in [24]. Moreover the computational complexity involved in finding the autocorrelation coefficients and in testing the suboptimal polynomials for stability is very high in [10].

To overcome these shortcomings, the present work, demonstrates a new way of form-preserving transformation concerning NSHP, to convert the 2D NSHP polynomial into 1D polynomial. The emphasis here is that the presented transformation when applied to NSHP will lead to optimal LSI. If one attempts to use this transformation for firstquadrant polynomial, this transformation will result in suboptimal LSI and, hence, results into unstable PLSI. Although QP filters form a subset of the more general NSHP filters, the stability requirement for NSHP and QP are different. A comparison on the stability of 2D QP and NSHP PLSI polynomials can be found in [5].

This section, demonstrates a new way of form-preserving transformation concerning NSHP polynomials, which will be used to obtain the PLSI polynomial that correspond to the given unstable 2D NSHP polynomials in a simple manner. As mentioned earlier, PLSI method is used to stabilize the unstable 2D NSHP polynomial corresponding to the 2D NSHP filter. Some definitions and theorems have been introduced related to it.

Definition 8. A polynomial $A_{F}(z)=\sum_{k=0}^{N} a_{k} z^{k}$ is a formpreserving $1 \mathrm{D}$ polynomial with respect to a $2 \mathrm{D}$ polynomial 
$A\left(z_{1}, z_{2}\right)=\sum_{R+\oplus}^{M} \sum_{R+\oplus}^{M} a_{m n} z_{1}^{m} z_{2}^{n}$ if for every integer set $(m, n)$ in $A\left(z_{1}, z_{2}\right)$ there exists a unique $k$ in $A_{F}(z)$ such that $a_{k}=$ $a_{m n}$.

Definition 9. Given a finite length discrete sequence of the form $\left\{a_{0}, a_{1}, a_{2}, \ldots, a_{k}\right\}$. The autocorrelation coefficients $\gamma_{i}$ 's of the sequence is defined as $\gamma_{i}=\sum_{j=0}^{k} a_{j} a_{j+i}$, where $i=$ $0,1,2, \ldots, k$, (see [25]).

This leads to introduce the following new theorem.

Theorem 1. If $A\left(z_{1}, z_{2}\right)=\sum_{R+\oplus}^{N} \sum_{R+\oplus}^{N} a_{m n} z_{1}^{m} z_{2}^{n}$ is a $2 D$ NSHP polynomial of degree $N$, then its form-preserving $1 D$ polynomial $A_{F}(z)=\left.A\left(z_{1} z_{2}\right)\right|_{z_{2}=z} ^{z_{1}=z^{L}}$, when $L=2 N+1$, will be nonlacuncary, and it will have the same autocorrelation coefficients as the polynomial $A\left(z_{1}, z_{2}\right)$.

Proof. For $A_{F}(z)$ to be a form-preserving 1D polynomial with respect to a $2 \mathrm{D}$ NSHP polynomial, $A\left(z_{1}, z_{2}\right)$ the number of distinct terms in these two polynomials should be the same. Let the 2D NSHP polynomial be of degree $N$.

From the nature of NSHP polynomials, the number of distinct terms in $A_{F}(z)$ is $N L+2 N+1$. Similarly, the number of distinct terms in $A\left(z_{1}, z_{2}\right)=(N+1)(2 N+1)$. To find the value of $L$, equate the number of terms in $A_{F}(z)$ and $A\left(z_{1}, z_{2}\right)$ :

$$
\begin{aligned}
N L+2 N+1 & =(N+1)(2 N+1), \\
N L+2 N+1 & =2 N N+2 N+N+1 \\
N L= & 2 N N+N \\
L & =2 N+1
\end{aligned}
$$

Thus the proof.

Let $A\left(z_{1}, z_{2}\right)=\sum_{R+\oplus}^{1} \sum_{R+\oplus}^{1} a_{m n} z_{1}^{m} z_{2}^{n}$ be a $2 \mathrm{D}$ NSHP polynomial of first degree. This 2D NSHP polynomial can be written as follows:

$$
A\left(z_{1}, z_{2}\right)=a_{00}+a_{01} z_{2}+a_{10} z_{1}+a_{11} z_{1} z_{2}+a_{1-1} z_{1} z_{2}^{-1}
$$

This imply to obtain the form-preserving $1 \mathrm{D}$ polynomial $A_{F}(z)$ of (19) with the form-preserving transformation $\left.A\left(z_{1}, z_{2}\right)\right|_{z_{2}=z} ^{z_{1}=z^{L}}$, when $L=2 N+1$ as follows:

$$
\begin{aligned}
& A_{F}(z)=A\left(z^{2 N+1}, z\right)=A\left(z^{3} z\right), \\
& A_{F}(z)=a_{00}+a_{01} z+a_{10} z^{3}+a_{11} z^{4}+a_{1-1} z^{2} .
\end{aligned}
$$

Equation (20) can be rewritten as below:

$$
A_{F}(z)=a_{00}+a_{01} z+a_{1-1} z^{2}+a_{10} z^{3}+a_{11} z^{4} .
$$

From (19) and (21), it is evident that the number of distinct terms in the $2 \mathrm{D}$ polynomial $A\left(z_{1}, z_{2}\right)$ is the same as the number of distinct terms in the form-preserving 1D polynomial $A_{F}(z)$ and in view of Theorem 1, the autocorrelation coefficients $\gamma_{i}$ 's of (19) and (21) are the same. They are as follows:

$$
\begin{gathered}
a_{00}^{2}+a_{01}^{2}+a_{1-1}^{2}+a_{10}^{2}+a_{11}^{2}=\gamma_{0}, \\
a_{00} a_{01}+a_{01} a_{1-1}+a_{1-1} a_{10}+a_{10} a_{11}=\gamma_{1}, \\
a_{00} a_{1-1}+a_{01} a_{10}+a_{10} a_{1-1}=\gamma_{2}, \\
a_{00} a_{10}+a_{01} a_{11}=\gamma_{3}, \\
a_{00} a_{11}=\gamma_{4} .
\end{gathered}
$$

Obviously from (21) the form-preserving 1D polynomial obtained from $A\left(z_{1}, z_{2}\right)$ with $L=(2 N+1)$ is nonlacunary (i.e., no missing powers of $z$ ). Therefore any positive integer value of $L>(2 N+1)$ will also result in form-preserving 1D polynomial, having the same autocorrelation coefficients as that of the given 2D polynomial, but the resulting $1 \mathrm{D}$ formpreserving polynomial will be definitely lacunary.

Before discussing the existing theorems related to stability, it is important to discover the relationship between stability and causality.

Stability does not include causality and anticausality. A causal system is stable, when all its poles lie inside the unit circle, the zeros are irrelevant. Likewise an anticausal system is stable, when all its poles lie outside the unit circle, again the zeros are irrelevant. Those systems that have poles right on the unit circle or called marginally stable. Causality is often a desirable constraint to impose in designing 1D systems. An anticausal system would require delay, which is undesirable in such applications as real-time speech processing. In typical 2D signal processing applications such as image processing, causality has little importance. At any given time, a complete frame of an image may be available for processing, and it may be processed from left to right, from top to bottom, or in any direction one chooses.

At this point, this paper defines the existing theorems related to stability in which $1 \mathrm{D}$ and $2 \mathrm{D} Z$-transforms are defined with positive powers of $Z$.

Theorem 2. Given a transfer function of certain 1D LSI system, $H(z)=1 / A(z)$. A polynomial $A(z)$ is stable if and only if

$$
A(z) \neq 0, \quad|z| \leq 1
$$

(see [18]). The condition as in (23) states that a $1 D$ polynomial is stable, if and only if, all its zeros lie outside the unit circle.

Theorem 3. Given a transfer function of some 2D LSI system, $H\left(z_{1}, z_{2}\right)=1 / A\left(z_{1}, z_{2}\right)$. A polynomial $A\left(z_{1}, z_{2}\right)$ is stable if and only if

$$
A\left(z_{1}, z_{2}\right) \neq 0, \quad \text { for } \bar{U}^{2}
$$

(see [18]).

Theorem 4. If $A^{\prime}\left(z_{1}, z_{2}\right)$ is a PLSI polynomial of $A\left(z_{1}, z_{2}\right)$, then $A_{F}^{\prime}(z)$ is the LSI polynomial of $A_{F}(z)$. 
Proof. The proof of this theorem derives from the definitions, and the method of obtaining PLSI polynomials discussed in Section 3. Initially, it is assumed that $A^{\prime}\left(z_{1}, z_{2}\right)$ is a PLSI of $A\left(z_{1}, z_{2}\right)$. The $2 \mathrm{D}$ polynomial $C\left(z_{1}, z_{2}\right)$ as given in (12), and its form-preserving 1D polynomial $C_{F}(z)$ will have the same coefficients. Therefore, the error function $E$ in (13) will be the same for both polynomials, and that results in the same set of linear equations $\bar{T} \overline{a^{\prime}}=\bar{a}$ as given in (15) and hence the coefficients of the polynomial $A_{F}^{\prime}(z)$ will turn out to be the same as those of $A^{\prime}\left(z_{1}, z_{2}\right)$. Hence $A_{F}^{\prime}(z)$ is the LSI of $A_{F}(z)$. The converse of Theorem 4 is also true.

This paper now introduces a new stability theorem. Theorems 1 and 4 are the basis for Theorem 5 .

Theorem 5. A 2D NSHP, PLSI polynomial $A^{\prime}\left(z_{1}, z_{2}\right)=$ $\sum_{R+\oplus}^{N} \sum_{R+\oplus}^{N} a_{m n}^{\prime} z_{1}^{m} z_{2}^{n}$ of degree $N$, is stable, if and only if, the $1 D$ form-preserving polynomial $A_{F}^{\prime}(z)$ obtained using the formpreserving transformation, $A^{\prime}\left(z^{2 N+1}, z\right)$ is stable.

Proof. The necessary and sufficient conditions for $A^{\prime}\left(z_{1}, z_{2}\right)$ to be stable is that [6]

$$
\begin{aligned}
& \text { (i) } A^{\prime}(z, z) \neq 0 \quad \text { when }|z| \leq 1 \text {; } \\
& \text { (ii) } A^{\prime}\left(z_{1}, z_{2}\right) \neq 0 \text { on } T^{2} .
\end{aligned}
$$

The new set of stability conditions equivalent to (25a) by applying homomorphic transformation [26] is stated as follows:

$$
\begin{aligned}
& \text { (i) } A^{\prime}\left(z^{l_{1}}, z^{l_{2}}\right) \neq 0 \text { when }|z| \leq 1 \text {; } \\
& \text { (ii) } A^{\prime}\left(z_{1}, z_{2}\right) \neq 0 \text { on } T^{2} \text {. }
\end{aligned}
$$

For some $l_{1}, l_{2} \in z^{+}$, where $z^{+}$denotes the positive integers [26]. It has been shown in [20] that the PLSI polynomial $A^{\prime}\left(z_{1}, z_{2}\right)$ of any $2 \mathrm{D}$ polynomial $A\left(z_{1}, z_{2}\right)$ devoid on $T^{2}$ itself. This satisfies the condition (ii) of (25b). Now we have to show that the condition (i) of (25b) is fullfilled. From Theorem $4, A_{F}^{\prime}(z)$ is the LSI of $A_{F}(z)$, and from Theorem 1, $A_{F}^{\prime}(z)$, which is obtained with the form-preserving transformation $z_{1}=z^{L}, z_{2}=z$ when $L=2 N+1$ has the same number of terms as in $A^{\prime}\left(z_{1}, z_{2}\right)$, and the autocorrelation coefficients of both $A_{F}^{\prime}(z)$ and $A^{\prime}\left(z_{1}, z_{2}\right)$ are the same. Obviously, only with this form-preserving transformation, the resulting 1-D LSI polynomial $A_{F}^{\prime}(z)$ is nonlacunary, and optimal while 1D LSI polynomial resulting from $L>2 N+1$ will definitely be lacunary, and suboptimal. It is an established fact that the optimal 1D LSI polynomial $A_{F}^{\prime}(z)$ is stable [25]. The formpreserving $1 \mathrm{D}$ polynomial, $A_{F}^{\prime}(z)=\left.A^{\prime}\left(z^{l_{1}}, z^{l_{2}}\right)\right|_{l_{2}=1} ^{l_{1}=2 N+1}$ is thus stable. Therefore, condition (i) of (25b) is also fulfilled. So, the PLSI polynomial $A^{\prime}\left(z_{1}, z_{2}\right)$ is stable. This demonstrates Theorem 5.

In the process of forming the PLSI polynomial corresponding to the given $2 \mathrm{D}$ NSHP polynomial, formpreserving transformation is used to convert the 2D NSHP polynomial into 1D and, then, obtain the 1D LSI of the formpreserving $1 \mathrm{D}$ polynomial. The PLSI can then be formed from 1D LSI.
From Theorems 4 and 5, it is obvious that, to prove PLSI polynomial $A^{\prime}\left(z_{1}, z_{2}\right)$ is stable, it is enough to demonstrate its form-preserving 1D polynomial, $A_{F}^{\prime}(z)$ with the transformation, $A^{\prime}\left(z^{2 N+1}, z\right)$ is stable.

\section{Numerical Examples}

This section, focuses on some numerical examples for stabilizing 2D NSHP polynomials of first and second degree. The proposed theorems and procedures are applied to form the PLSI polynomials in the examples provided by the literature [10], and demonstrate that, the proposed theorems and procedures still result in a stable PLSI polynomial as with the existing method in the literature, but with reduced complexity.

Example 1 (Polynomial of first degree). Consider the following 2D NSHP polynomial of first degree:

$$
A\left(z_{1}, z_{2}\right)=0.9 z_{1} z_{2}^{-1}+0.3+0.6 z_{2}+0.6 z_{1}+0.8 z_{1} z_{2}
$$

(see [10]). The form-preserving $1 \mathrm{D}$ polynomial $A_{F}(z)$ of $A\left(z_{1}, z_{2}\right)$ is obtained using the new way of form-preserving transformation (from Theorem 1)

$$
A_{F}(z)=A\left(z^{2 N+1}, z\right)=A\left(z^{3} z\right) \quad(\text { since } N=1) .
$$

Thus,

$$
A_{F}(z)=0.8 z^{4}+0.6 z^{3}+0.9 z^{2}+0.6 z+0.3
$$

The roots of $A_{F}(z)$ are $0.9393,0.9393,0.6520$, and 0.6520 . From Theorem 2, therefore, the form-preserving 1D polynomial is unstable. Theorem 4 is applied to form the LSI polynomial of $A_{F}(z)$. The LSI of $A_{F}(z)$ is $A_{F}^{\prime}(z)$ and to find out $A_{F}^{\prime}(z)$, the autocorrelation coefficients of $A_{F}(z)$ are computed as follows:

$$
\gamma_{0}=2.26, \quad \gamma_{1}=1.74, \quad \gamma_{2}=1.35, \quad \gamma_{3}=0.66, \quad \gamma_{4}=0.24
$$

Now the normal equation is formed (15) as follows:

$$
\left[\begin{array}{lllll}
2.26 & 1.74 & 1.35 & 0.66 & 0.24 \\
1.74 & 2.26 & 1.74 & 1.35 & 0.66 \\
1.35 & 1.74 & 2.26 & 1.74 & 1.35 \\
0.66 & 1.35 & 1.74 & 2.26 & 1.74 \\
0.24 & 0.66 & 1.35 & 1.74 & 2.26
\end{array}\right] \times\left[\begin{array}{c}
a_{00}^{\prime} \\
a_{01}^{\prime} \\
a_{1-1}^{\prime} \\
a_{10}^{\prime} \\
a_{11}^{\prime}
\end{array}\right]=\left[\begin{array}{c}
0.3 \\
0 \\
0 \\
0 \\
0
\end{array}\right],
$$




$$
\begin{gathered}
{\left[\begin{array}{l}
a_{00}^{\prime} \\
a_{01}^{\prime} \\
a_{1-1}^{\prime} \\
a_{10}^{\prime} \\
a_{11}^{\prime}
\end{array}\right]=\left[\begin{array}{lllll}
2.26 & 1.74 & 1.35 & 0.66 & 0.24 \\
1.74 & 2.26 & 1.74 & 1.35 & 0.66 \\
1.35 & 1.74 & 2.26 & 1.74 & 1.35 \\
0.66 & 1.35 & 1.74 & 2.26 & 1.74 \\
0.24 & 0.66 & 1.35 & 1.74 & 2.26
\end{array}\right]^{-1} \times\left[\begin{array}{c}
0.3 \\
0 \\
0 \\
0 \\
0
\end{array}\right], \quad(31)} \\
{\left[\begin{array}{c}
a_{00}^{\prime} \\
a_{01}^{\prime} \\
a_{1-1}^{\prime} \\
a_{10}^{\prime} \\
a_{11}^{\prime}
\end{array}\right]=\left[\begin{array}{c}
0.3964 \\
-0.3078 \\
-0.1282 \\
0.1744 \\
-0.0100
\end{array}\right],} \\
A_{F}^{\prime}(z)=0.3964-0.3078 z-0.1282 z^{2}+0.1744 z^{3}-0.0100 z^{4} .
\end{gathered}
$$

The roots of $A_{F}^{\prime}$ are $16.5625,1.4479,1.2857$, and 1.2857. From Theorem 2, therefore the 1D LSI polynomial $A_{F}^{\prime}(z)$ corresponding to $A_{F}(z)$ is stable. It is interesting that, even though $2 \mathrm{D}$ to $1 \mathrm{D}$ transformations in general are irreversible (i.e., singular) in the case of form-preserving transformations, there exists a one-to-one mapping among the coefficients of the $2 \mathrm{D}$, and the transformed $1 \mathrm{D}$ polynomials, and the 2D polynomials can be constructed exactly from the $1 \mathrm{D}$ polynomial. Thus, the 2D PLSI corresponding to the 1D LSI (33) reads as follows:

$$
\begin{aligned}
A^{\prime}\left(z_{1}, z_{2}\right)= & 0.3964-0.3078 z_{2}+0.1744 z_{1} \\
& -0.0100 z_{1} z_{2}-0.1282 z_{1} z_{2}^{-1} .
\end{aligned}
$$

The PLSI polynomial demonstrated in (34) is stable in view of Theorem 5. This is in line with the result reported in [10].
Example 2 (Polynomial of second degree). Consider the following 2D NSHP polynomial of second degree:

$$
\begin{aligned}
A\left(z_{1}, z_{2}\right)= & 0.6+0.9 z_{2}+0.3 z_{2}^{2}+0.9 z_{1}+1.5 z_{1} z_{2}+0.9 z_{1} z_{2}^{2} \\
& +0.3 z_{1}^{2}+0.9 z_{1}^{2} z_{2}+0.6 z_{1}^{2} z_{2}^{2}+0.5 z_{1} z_{2}^{-1} \\
& +0.8 z_{1}^{2} z_{2}^{-1}+0.7 z_{1} z_{2}^{-2}+z_{1}^{2} z_{2}^{-2}
\end{aligned}
$$

(see [10]). The form-preserving $1 \mathrm{D}$ polynomial $A_{F}(z)$ of $A\left(z_{1}, z_{2}\right)$ is obtained using the new way of form-preserving transformation (from Theorem 1):

$$
\begin{aligned}
A_{F}(z)= & A\left(z^{2 N+1}, z\right)=A\left(z^{5}, z\right) \quad(\text { since } N=2) \\
A_{F}(z)= & 0.6+0.9 z+0.3 z^{2}+0.9 z^{5}+1.5 z^{6}+0.9 z^{7}+0.3 z^{10} \\
& +0.9 z^{11}+0.6 z^{12}+0.5 z^{4}+0.8 z^{9}+0.7 z^{3}+z^{8}
\end{aligned}
$$

This can be read as follows:

$$
\begin{aligned}
A_{F}(z)= & 0.6+0.9 z+0.3 z^{2}+0.7 z^{3}+0.5 z^{4}+0.9 z^{5}+1.5 z^{6} \\
& +0.9 z^{7}+z^{8}+0.8 z^{9}+0.3 z^{10}+0.9 z^{11}+0.6 z^{12}
\end{aligned}
$$

The roots of $A_{F}(z)$ are $1.0728,1.0728,0.9481,0.9481$, $1.0021,1.0021,0.9545,0.9545,1.3298,1.0892,1.0892$, and 0.6697. From Theorem 2, therefore, the form-preserving 1D polynomial is unstable. Theorem 4 is applied to form the LSI polynomial of $A_{F}(z)$. The LSI of $A_{F}(z)$ is $A_{F}^{\prime}(z)$ and to find out $A_{F}^{\prime}(z)$, the autocorrelation coefficients of $A_{F}(z)$ are computed as follows: $\gamma_{0}=8.77, \gamma_{1}=7.27, \gamma_{2}=6.57$, $\gamma_{3}=6.39, \gamma_{4}=5.27, \gamma_{5}=5.42, \gamma_{6}=4.43, \gamma_{7}=2.88$, $\gamma_{8}=2.34, \gamma_{9}=1.44, \gamma_{10}=1.17, \gamma_{11}=1.08, \gamma_{12}=0.36$.

Now form the normal equation (15) as follows:

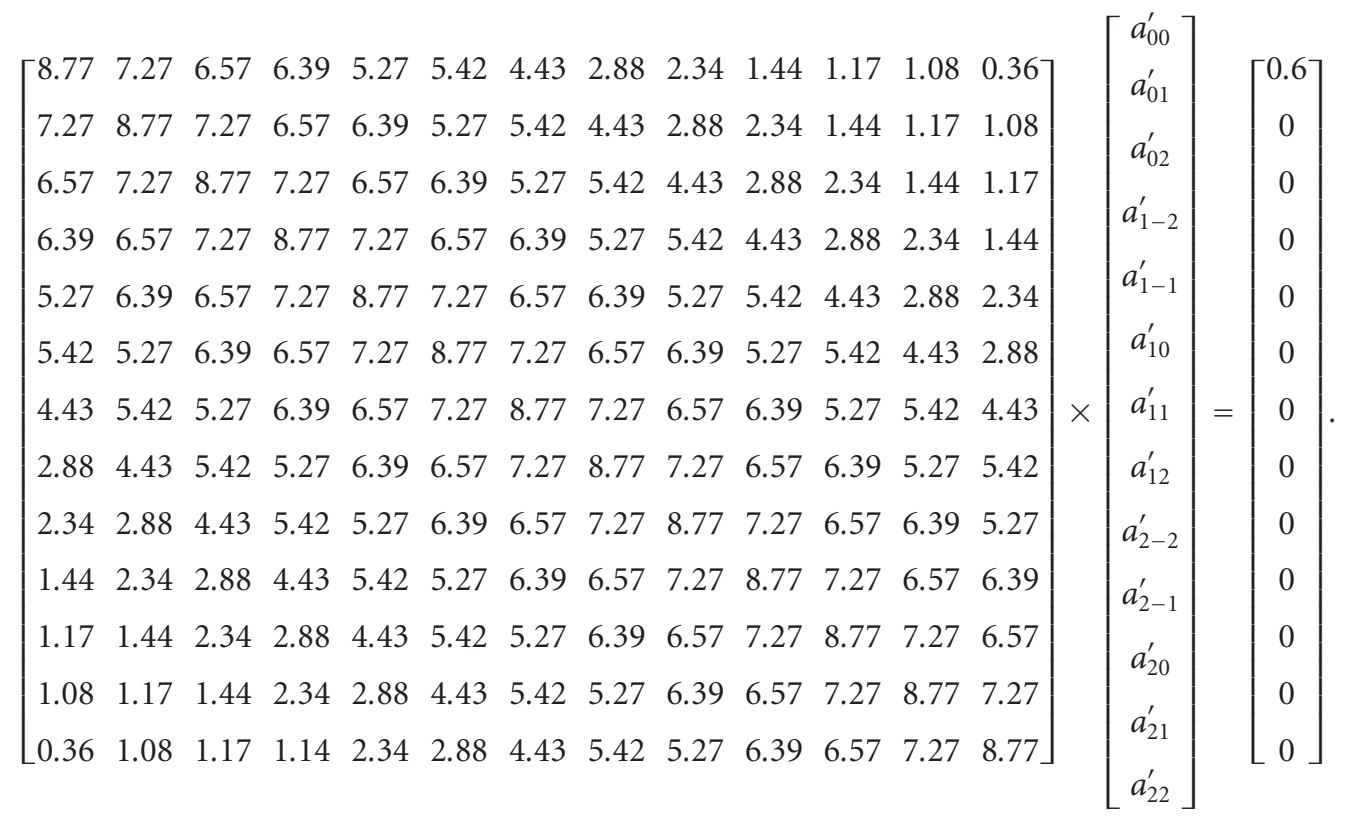


Solving (38) we get,

$$
\left[\begin{array}{l}
a_{00}^{\prime} \\
a_{01}^{\prime} \\
a_{02}^{\prime} \\
a_{1-2}^{\prime} \\
a_{1-1}^{\prime} \\
a_{10}^{\prime} \\
a_{11}^{\prime} \\
a_{12}^{\prime} \\
a_{2-2}^{\prime} \\
a_{2-1}^{\prime} \\
a_{20}^{\prime} \\
a_{21}^{\prime} \\
a_{22}^{\prime}
\end{array}\right]=\left[\begin{array}{c}
0.4231 \\
-0.3344 \\
0.0815 \\
-0.2717 \\
0.2325 \\
-0.3339 \\
0.1882 \\
-0.0001 \\
0.1563 \\
-0.0342 \\
0.0167 \\
-0.0488 \\
-0.0309
\end{array}\right] .
$$

Now, the PLSI $A_{F}^{\prime}(z)$ is

$$
\begin{aligned}
A_{F}^{\prime}(z)= & 0.4231-0.3344 z+0.0815 z^{2}-0.2717 z^{3}+0.2325 z^{4} \\
& -0.3339 z^{5}+0.1882 z^{6}-0.0001 z^{7}+0.1563 z^{8} \\
& -0.0342 z^{9}+0.0167 z^{10}-0.0488 z^{11}-0.0309 z^{12}
\end{aligned}
$$

The roots of $A_{F}^{\prime}(z)$ are as follows: $2.4606,1.1771,1.1771$, $1.1854,1.1854,1.4025,1.4025,1.0727,1.0727,1.0782$, 1.0782, and 1.0863. From Theorem 2, therefore, 1D LSI polynomial $A_{F}^{\prime}(z)$ corresponding to $A_{F}(z)$ is stable. The $2 \mathrm{D}$ PLSI obtained from LSI (40) is as follows:

$$
\begin{aligned}
A^{\prime}\left(z_{1}, z_{2}\right)= & 0.4231-0.3344 z_{2}+0.0815 z_{2}^{2}-0.3339 z_{1} \\
& +0.1882 z_{1} z_{2}-0.0001 z_{1} z_{2}^{2}+0.0167 z_{1}^{2} \\
& -0.0488 z_{1}^{2} z_{2}-0.0309 z_{1}^{2} z_{2}^{2}+0.2325 z_{1} z_{2}^{-1} \\
& -0.0342 z_{1}^{2} z_{2}^{-1}-0.2717 z_{1} z_{2}^{-2}+0.1563 z_{1}^{2} z_{2}^{-2} .
\end{aligned}
$$

The PLSI polynomial given in (41) is stable in view of Theorem 5. This result is also in line with the results reported in [10].

Example 3 (Polynomial of first degree). Consider the following 2D NSHP polynomial of first degree (random example):

$$
A\left(z_{1}, z_{2}\right)=0.2 z_{1} z_{2}^{-1}+0.1+0.7 z_{2}+0.9 z_{1}+0.4 z_{1} z_{2} .
$$

The form-preserving 1D polynomial $A_{F}(z)$ of $A\left(z_{1}, z_{2}\right)$ is obtained using the new way of form-preserving transformation (from Theorem 1):

$$
A_{F}(z)=A\left(z^{2 N+1}, z\right)=A\left(z^{3}, z\right) \quad(\text { since } N=1) .
$$

Thus,

$$
A_{F}(z)=0.4 z^{4}+0.9 z^{3}+0.2 z^{2}+0.7 z+0.1 .
$$

The roots of $A_{F}(z)$ are $2.3369,0.8584,0.8584$, and 0.1452 . From Theorem 2, therefore, the form-preserving 1D polynomial is unstable. The LSI of $A_{F}(z)$ is $A_{F}^{\prime}(z)$ and to find out $A_{F}^{\prime}(z)$, the autocorrelation coefficients of $A_{F}(z)$ are computed as follows:

$$
\gamma_{0}=1.51, \quad \gamma_{1}=0.75, \quad \gamma_{2}=0.73, \quad \gamma_{3}=0.37, \quad \gamma_{4}=0.04 .
$$

Now form the normal equation (15) as follows:

$$
\left[\begin{array}{ccccc}
1.51 & 0.75 & 0.73 & 0.37 & 0.04 \\
0.75 & 1.51 & 0.75 & 0.73 & 0.37 \\
0.73 & 0.75 & 1.51 & 0.75 & 0.73 \\
0.37 & 0.73 & 0.75 & 1.51 & 0.75 \\
0.04 & 0.37 & 0.73 & 0.75 & 1.51
\end{array}\right] \times\left[\begin{array}{c}
a_{00}^{\prime} \\
a_{01}^{\prime} \\
a_{1-1}^{\prime} \\
a_{10}^{\prime} \\
a_{11}^{\prime}
\end{array}\right]=\left[\begin{array}{c}
0.1 \\
0 \\
0 \\
0 \\
0
\end{array}\right],
$$

$$
\left[\begin{array}{c}
a_{00}^{\prime} \\
a_{01}^{\prime} \\
a_{1-1}^{\prime} \\
a_{10}^{\prime} \\
a_{11}^{\prime}
\end{array}\right]=\left[\begin{array}{ccccc}
1.51 & 0.75 & 0.73 & 0.37 & 0.04 \\
0.75 & 1.51 & 0.75 & 0.73 & 0.37 \\
0.73 & 0.75 & 1.51 & 0.75 & 0.73 \\
0.37 & 0.73 & 0.75 & 1.51 & 0.75 \\
0.04 & 0.37 & 0.73 & 0.75 & 1.51
\end{array}\right]^{-1} \times\left[\begin{array}{c}
0.1 \\
0 \\
0 \\
0 \\
0
\end{array}\right],
$$

$$
\begin{gathered}
{\left[\begin{array}{c}
a_{00}^{\prime} \\
a_{01}^{\prime} \\
a_{1-1}^{\prime} \\
a_{10}^{\prime} \\
a_{11}^{\prime}
\end{array}\right]=\left[\begin{array}{c}
0.1065 \\
-0.0368 \\
-0.0476 \\
0.0011 \\
0.0287
\end{array}\right],} \\
A_{F}^{\prime}(z)=0.1065-0.0368 z-0.0476 z^{2}+0.0011 z^{3}+0.0287 z^{4} .
\end{gathered}
$$

The roots of $A_{F}^{\prime}(z)$ are 1.4952, 1.4952, 1.2884, and 1.2884. From Theorem 2, therefore, 1D LSI polynomial $A_{F}^{\prime}(z)$ corresponding to $A_{F}(z)$ is stable. Then, the 2D PLSI is obtained in relationship to the 1D LSI of (49) as follows:

$$
\begin{aligned}
A^{\prime}\left(z_{1}, z_{2}\right)= & 0.1065-0.0368 z_{2}+0.0011 z_{1} \\
& -0.0287 z_{1} z_{2}-0.0476 z_{1} z_{2}^{-1} .
\end{aligned}
$$

The PLSI polynomial given in (50) is stable in view of Theorem 5 .

It is evident, from the above three numerical examples, discussed in this section that, the PLSI polynomial obtained with the new way of form-preserving transformation always results in a stable system. 


\section{Lagrange Multiplier Method for Testing Stability}

In all the three numerical examples discussed, in the preceding section, the stability of the form-preserving 1D LSI was tested using root finding method. The stability of only first-, and second-degree LSI polynomials was dealt with. In order to generalize the validity for any degree, a theoretical method of testing the stability of the form-preserving ID LSI based on Lagrange Multipliers Method is introduced.

It is well known that, in the process of forming LSI polynomial, the minimum error $E$ as in (7) is $E=1-$ $a_{0} a_{0}^{\prime}$, where $a_{0}$ and $a_{0}^{\prime}$ are the constant terms in the original unstable polynomial, and LSI polynomial, respectively, [10, 11]. It is demonstrated in [27] that, for a polynomial of degree $N$ there are in total $2^{N}$ polynomials, including the given polynomial, which will have the same autocorrelation coefficients as the given polynomial and hence the same magnitude spectra, and out of which, only one polynomial is stable for which the constant term is highest in order to achieve minimum error. Since $A_{F}^{\prime}(z)$ is the form-preserving $1 \mathrm{D}$ polynomial of $A^{\prime}\left(z_{1}, z_{2}\right)$, to demonstrate that the PLSI polynomial $A^{\prime}\left(z_{1}, z_{2}\right)$ is stable, it is enough that the constant term of the form-preserving $1 \mathrm{D}$ polynomial, $A_{F}^{\prime}(z)$ is the highest. So we only test the 1D LSI $A_{F}^{\prime}(z)$ for stability.

Let $A_{F}^{\prime \prime}(z)$ be the stable version of $A_{F}^{\prime}(z)$. In this method, one has to maximize the function $f$ as

$$
f=a_{00}^{\prime \prime},
$$

satisfying the constraints $g_{i}$ given as

$$
g_{i}=\sum_{r=0}^{N} a_{r}^{\prime \prime} a_{r+s}^{\prime \prime}-\gamma_{s}=0, \quad s=0,1,2, \ldots, N
$$

where

$$
\gamma_{S}=\sum_{r=0}^{N} a_{r}^{\prime} a_{r+s}^{\prime}, \quad s=0,1,2, \ldots, N
$$

That is,

$$
g_{i}=0, \quad i=0,1,2, \ldots, N
$$

Then, the Lagrange function is formed, $L\left(a_{00}^{\prime \prime}, \lambda_{j}\right)=f+$ $\sum_{j=0}^{N} \lambda_{j} g_{j}$, where $\lambda_{j}$ 's are the Lagrange multipliers. Then form

$$
\begin{aligned}
& \frac{\partial L\left(a_{00}^{\prime \prime}, \lambda_{j}\right)}{\partial a_{00}^{\prime \prime}}=0, \\
& \frac{\partial L\left(a_{00}^{\prime \prime}, \lambda_{j}\right)}{\partial \lambda_{j}}=0, \quad j=0,1,2, \ldots, N .
\end{aligned}
$$

Equation (56) is called Lagrange equation.

In this method, if the number of unknowns $\left(a_{m n}^{\prime \prime}\right.$ 's and $\lambda_{j}$ 's) is greater than the number of constraint equations (55) and (56), then, the solution may exist for the set of equations and the optimum can be obtained for its constant term $a_{00}^{\prime}$. If so, the form-preserving 1D LSI will be stable and hence the
PLSI. On the other hand, if the number of unknowns is less than the number of constraint equations, the solution may not exist, and optimum may not be obtained for its constant term $a_{00}^{\prime}$. In this case, the PLSI may be unstable.

In the computer-aided optimization method of solving nonlinear equations, one will be assured of a real solution if the total number of unknowns, namely, $a_{m n}^{\prime \prime}$ 's and $\lambda_{j}$ 's is greater than the number of equations by at least one. Our interest in this section is in establishing theoretically the fact that, an optimum solution exists for the set of nonlinear equations in the maximization process. This ensures the stability of the LSI polynomial and hence PLSI polynomial. On the other hand, if the number of unknowns is less than the number of nonlinear equations, in the computer-aided optimization, and since the programmer has no degree of freedom, sometimes it may not give us any real solution at all. If this is the case, the PLSI will not be stable.

If the nonlinear equations given in (55) and (56) are solvable and if the solution with all $\lambda_{j}$ 's positive exists, then the necessary condition for the existence of the optimum is satisfied, and the real optimum for $a_{00}^{\prime \prime}$ in the maximization process using Lagrange multiplier method will be reached. If so resulting LSI and PLSI polynomial will be definitely stable.

The following examples clearly illustrate the application of Lagrange multiplier method in testing the stability of LSI and hence PLSI polynomial.

Example 4 (Polynomial of first degree). Consider a 2D PLSI polynomial of first degree:

$$
A^{\prime}\left(z_{1}, z_{2}\right)=a_{00}^{\prime}+a_{01}^{\prime} z_{2}+a_{10}^{\prime} z_{1}+a_{11}^{\prime} z_{1} z_{2}+a_{1-1}^{\prime} z_{1} z_{2}^{-1}
$$

and test the stability of (57) applying the proposed and existing methods.

New Method. The form-preserving 1D polynomial of $A^{\prime}\left(z_{1}, z_{2}\right)$ in (57) with the proposed form-preserving transformation $A_{F}^{\prime}(z)=A^{\prime}\left(z^{2 N+1} z\right)=A^{\prime}\left(z^{3} z\right)$ ( since $N=1$ ) is as follows:

$$
A_{F}^{\prime}(z)=a_{00}^{\prime}+a_{01}^{\prime} z+a_{1-1}^{\prime} z^{2}+a_{10}^{\prime} z^{3}+a_{11}^{\prime} z^{4}
$$

Let

$$
A_{F}^{\prime \prime}(z)=a_{00}^{\prime \prime}+a_{01}^{\prime \prime} z+a_{1-1}^{\prime \prime} z^{2}+a_{10}^{\prime \prime} z^{3}+a_{11}^{\prime \prime} z^{4}
$$

be the stable version of polynomial $A_{F}^{\prime}(z)$. The autocorrelation constraint equations of (59) are as follows:

$$
\begin{gathered}
a_{00}^{\prime \prime 2}+a_{01}^{\prime \prime 2}+a_{1-1}^{\prime \prime 2}+a_{10}^{\prime \prime 2}+a_{11}^{\prime \prime 2}=\gamma_{0}^{\prime \prime}, \\
a_{00}^{\prime \prime} a_{01}^{\prime \prime}+a_{01}^{\prime \prime} a_{1-1}^{\prime \prime}+a_{1-1}^{\prime \prime} a_{10}^{\prime \prime}+a_{10}^{\prime \prime} a_{11}^{\prime \prime}=\gamma_{1}^{\prime \prime}, \\
a_{00}^{\prime \prime} a_{1-1}^{\prime \prime}+a_{01}^{\prime \prime} a_{10}^{\prime \prime}+a_{10}^{\prime \prime} a_{1-1}^{\prime \prime}=\gamma_{2}^{\prime \prime}, \\
a_{00}^{\prime \prime} a_{10}^{\prime \prime}+a_{01}^{\prime \prime} a_{11}^{\prime \prime}=\gamma_{3}^{\prime \prime}, \\
a_{00}^{\prime \prime} a_{11}^{\prime \prime}=\gamma_{4}^{\prime \prime} .
\end{gathered}
$$


This implies that, at the end of maximization process, five autocorrelation constraint equations are reached as in (60), and one Lagrange equation as in (56) totalling six nonlinear equations. There are $5 a_{m n}^{\prime \prime}$ 's, and $5 \lambda_{j}$ 's as unknowns, totalling 10 unknowns, which can be easily solved, and the optimum $a_{00}^{\prime \prime}$ exists. Therefore, in this case the LSI as in (58) and PLSI as in (57) will be definitely stable.

Existing Method. The form-preserving 1D polynomial of $A^{\prime}\left(z_{1}, z_{2}\right)$ with the existing form-preserving transformation $[10]$ is as follows:

$$
\begin{aligned}
& A_{F}^{\prime}(z)=A^{\prime}\left(z^{4 N+1} z\right)=A^{\prime}\left(z^{5} z\right) \\
& A_{F}^{\prime}(z)=a_{00}^{\prime}+a_{01} z+0+0+a_{1-1} z^{4}+a_{10} z^{5}+a_{11} z^{6}
\end{aligned}
$$

Let $A_{F}^{\prime \prime}(z)$ be the stable version of (62). The autocorrelation coefficients of (62) are as follows:

$$
\begin{gathered}
a_{00}^{\prime \prime 2}+a_{01}^{\prime \prime 2}+a_{1-1}^{\prime \prime 2}+a_{10}^{\prime \prime 2}+a_{11}^{\prime \prime 2}=\gamma_{0}^{\prime \prime}, \\
a_{00}^{\prime \prime} a_{01}^{\prime \prime}+a_{1-1}^{\prime \prime} a_{10}^{\prime \prime}+a_{10}^{\prime \prime} a_{11}^{\prime \prime}=\gamma_{1}^{\prime \prime}, \\
a_{1-1}^{\prime \prime} a_{11}^{\prime \prime}=\gamma_{2}^{\prime \prime *}, \\
a_{01}^{\prime \prime} a_{1-1}^{\prime \prime}=\gamma_{3}^{\prime \prime *}, \\
a_{00}^{\prime \prime} a_{1-1}^{\prime \prime}+a_{01}^{\prime \prime} a_{10}^{\prime \prime}=\gamma_{4}^{\prime \prime}, \\
a_{00}^{\prime \prime} a_{10}^{\prime \prime}+a_{01}^{\prime \prime} a_{11}^{\prime \prime}=\gamma_{5}^{\prime \prime}, \\
a_{00}^{\prime \prime} a_{11}^{\prime \prime}=\gamma_{6}^{\prime \prime} .
\end{gathered}
$$

In $(63), *$ indicates the autocorrelation constraint equations that do not contain $a_{00}^{\prime \prime}$.

The autocorrelation constraint equations given in (63) are seven in number. It may be noted that, two of these equations do not contain the constant coefficient $a_{00}^{\prime \prime}$. In this example, the total number of nonlinear equations is eight including the Lagrange equation. The total unknowns will be $5+5=10$. In this, the number of $\lambda_{j}$ 's have been considered as only five because $\lambda_{j}$ 's do not have to be assigned for the nonlinear equations that do not contain $a_{00}^{\prime \prime}$. Thus, there are 10 unknowns and eight equations. With the existing method also, the 1D LSI (62) PLSI (57) is found stable, but with the new method proposed in this paper, the number of equations is only six.

\section{Example 5 (Polynomial of second degree).}

New Method. Let $A^{\prime}\left(z_{1}, z_{2}\right)=\sum_{R+\oplus}^{2} \sum_{R+\oplus}^{2} a_{m n}^{\prime} z_{1}^{m} z_{2}^{n}$ be a second-degree polynomial, which can be expanded as

$$
\begin{aligned}
A^{\prime}\left(z_{1}, z_{2}\right)= & a_{00}^{\prime}+a_{01}^{\prime} z_{2}+a_{02}^{\prime} z_{2}^{2}+a_{10}^{\prime} z_{1}+a_{11}^{\prime} z_{1} z_{2}+a_{12}^{\prime} z_{1} z_{2}^{2} \\
& +a_{20}^{\prime} z_{1}^{2}+a_{21}^{\prime} z_{1}^{2} z_{2}+a_{22}^{\prime} z_{1}^{2} z_{2}^{2}+a_{1-1}^{\prime} z_{1} z_{2}^{-1} \\
& +a_{2-1}^{\prime} z_{1}^{2} z_{2}^{-1}+a_{1-2}^{\prime} z_{1} z_{2}^{-2}+a_{2-2}^{\prime} z_{1}^{2} z_{2}^{-2} .
\end{aligned}
$$

The form-preserving $1 \mathrm{D}$ polynomial $A_{F}^{\prime}(z)$ obtained with $L=2 N+1$, in the PLSI polynomial $A^{\prime}\left(z_{1}, z_{2}\right)$ is

$$
\begin{aligned}
A_{F}^{\prime}(z)= & A^{\prime}\left(z^{2 N+1}, z\right)=A^{\prime}\left(z^{5} z\right)=a_{00}^{\prime}+a_{01}^{\prime} z+a_{02}^{\prime} z^{2}+a_{10}^{\prime} z^{5} \\
& +a_{11}^{\prime} z^{6}+a_{12}^{\prime} z^{7}+a_{20}^{\prime} z^{10}+a_{21}^{\prime} z^{11}+a_{22}^{\prime} z^{12} \\
& +a_{1-1}^{\prime} z^{4}+a_{2-1}^{\prime} z^{9}+a_{1-2}^{\prime} z^{3}+a_{2-2}^{\prime} z^{8} .
\end{aligned}
$$

This can be written as follows:

$$
\begin{aligned}
A_{F}^{\prime}(z)= & a_{00}^{\prime}+a_{01}^{\prime} z+a_{02}^{\prime} z^{2}+a_{1-2}^{\prime} z^{3}+a_{1-1}^{\prime} z^{4}+a_{10}^{\prime} z^{5}+a_{11}^{\prime} z^{6} \\
& +a_{12}^{\prime} z^{7}+a_{2-2}^{\prime} z^{8}+a_{2-1}^{\prime} z^{9}+a_{20}^{\prime} z^{10}+a_{21}^{\prime} z^{11}+a_{22}^{\prime} z^{12} .
\end{aligned}
$$

This paper uses Lagrange multiplier method as in Example 4, to test whether the LSI, and whether PLSI in Example 5 is stable or not. At the end of maximization process, we will arrive at a total of 14 equations including one Lagrange equation is reached at. There are $13 a_{m n}^{\prime \prime}$ 's and $13 \lambda_{j}$ 's as unknowns totalling 26 unknowns, which can be easily solved, and the optimum $a_{00}^{\prime \prime}$ exists. Therefore, in this case, also the PLSI will be definitely stable.

Existing Method. The form-preserving 1D polynomial $A_{F}^{\prime}(z)$ obtained with $L=4 N+1$, in the PLSI polynomial $A^{\prime}\left(z_{1}, z_{2}\right)$ is

$$
\begin{aligned}
A_{F}^{\prime}(z)= & A^{\prime}\left(z^{4 N+1}, z\right)=A^{\prime}\left(z^{9} z\right)=a_{00}^{\prime}+a_{01}^{\prime} z+a_{02}^{\prime} z^{2}+a_{10}^{\prime} z^{9} \\
& +a_{11}^{\prime} z^{10}+a_{12}^{\prime} z^{11}+a_{20}^{\prime} z^{18}+a_{21}^{\prime} z^{19}+a_{22}^{\prime} z^{20} \\
& +a_{1-1}^{\prime} z^{8}+a_{2-1}^{\prime} z^{17}+a_{1-2}^{\prime} z^{7}+a_{2-2}^{\prime} z^{16} .
\end{aligned}
$$

This can be written as follows:

$$
\begin{aligned}
A_{F}^{\prime}(z)= & A^{\prime}\left(z^{4 N+1} z\right)=A^{\prime}\left(z^{9} z\right)=a_{00}^{\prime}+a_{01}^{\prime} z+a_{02}^{\prime} z^{2}+0 z^{3} \\
& +0 z^{4}+0 z^{5}+0 z^{6}+a_{1-2}^{\prime} z^{7}+a_{1-1}^{\prime} z^{8} \\
& +a_{10}^{\prime} z^{9}+a_{11}^{\prime} z^{10}+a_{12}^{\prime} z^{11}+0 z^{12}+0 z^{13} \\
& +0 z^{14}+0 z^{15}+a_{2-2}^{\prime} z^{16}+a_{2-1}^{\prime} z^{17} \\
& +a_{20}^{\prime} z^{18}+a_{21}^{\prime} z^{19}+a_{22}^{\prime} z^{20} .
\end{aligned}
$$

The autocorrelation constraint equations given in (68) are 21 in number. It may be noted that, eight of these equations do not contain the constant coefficient $a_{00}^{\prime \prime}$. In this example, the total number of nonlinear equations is 22 including the Lagrange equation. The total unknowns will be $13+13=26$. The number of $\lambda_{j}$ 's is only 13 because, it is not necessary to assign $\lambda_{j}$ 's for the nonlinear equations that do not contain $a_{00}^{\prime \prime}$. Thus, there are 26 unknowns and 22 equations. With the existing method also, the PLSI is found stable, but, with the new method proposed in this paper, the number of equations is only 14 . 
TABLE 1: Computational complexity.

\begin{tabular}{|c|c|c|c|c|c|c|}
\hline \multirow[t]{2}{*}{$\begin{array}{l}\text { Degree of } \\
\text { polynomial } \\
(N)\end{array}$} & \multicolumn{2}{|c|}{$\begin{array}{c}\text { Total number of } \\
\text { nonlinear equations } \\
\text { (including Lagrange equation) }\end{array}$} & \multicolumn{2}{|c|}{$\begin{array}{c}\text { Total number of } \\
\text { unknowns } \\
a_{m n}^{\prime \prime}+\lambda_{j}\end{array}$} & \multicolumn{2}{|c|}{$\begin{array}{c}\text { Dimension of } \bar{T} \text { matrix in } \\
\text { normal equation }\end{array}$} \\
\hline & Existing method & $\begin{array}{l}\text { New (proposed) } \\
\text { method }\end{array}$ & Existing method & $\begin{array}{l}\text { New (proposed) } \\
\text { method }\end{array}$ & Existing method & $\begin{array}{l}\text { New (proposed) } \\
\text { method }\end{array}$ \\
\hline 1 & 8 & 5 & 10 & 10 & $7 \times 7$ & $5 \times 5$ \\
\hline 5 & 112 & 61 & 122 & 122 & $111 \times 111$ & $61 \times 61$ \\
\hline 10 & 422 & 221 & 442 & 442 & $421 \times 421$ & $221 \times 221$ \\
\hline 15 & 932 & 481 & 962 & 962 & $931 \times 931$ & $481 \times 481$ \\
\hline 20 & 1642 & 841 & 1682 & 1682 & $1641 \times 1641$ & $841 \times 841$ \\
\hline
\end{tabular}

Example 6 (Polynomial of degree $N$ ). This example generalizes the results for the PLSI polynomial of degree $N$. Compute the number of unknowns and nonlinear equations applying the new proposed method and existing method. Results will be as follows.

New Method. With the proposed method, to test the LSI and PLSI polynomial of $N$ th degree for stability using Lagrange multiplier method, the following figures in the optimization process will be arrived at. The total number of nonlinear equations will be $2 N^{2}+2 N+1$, and the total number of unknowns will be $\left(2 N^{2}+2 N+1\right)+\left(2 N^{2}+2 N+1\right)=$ $4 N^{2}+4 N+2$. Since, $4 N^{2}+4 N+2>2 N^{2}+2 N+1$, the number of unknowns, will be always more than the number of equations, and these set of equations can easily be solved, and $a_{00}^{\prime \prime}$ exists. So the resulting PLSI using the new method will be always stable.

Existing Method. With the existing method, to test whether the LSI, and PLSI polynomial of Nth degree for stability, using Lagrange multiplier method, the following figures in the optimization process will be arrived at. The total number of nonlinear equations will be $4 N^{2}+2 N+1$. However, the number of unknowns will be $\left(2 N^{2}+2 N+1\right)+\left(2 N^{2}+2 N+1\right)=$ $4 N^{2}+4 N+2$. In the existing method, the total number of nonlinear equations involved is $4 N^{2}+2 N+1$, which is nearly double the number of nonlinear equations as in the new method proposed in this paper. In addition, for the $N$ th degree polynomial, with the existing method, size of $\bar{T}$ matrix (entries of autocorrelation coefficients) in the normal equation is $\left(4 N^{2}+2 N+1\right) \times\left(4 N^{2}+2 N+1\right)$, while with the new method, size of $\bar{T}$ matrix is only $\left(2 N^{2}+2 N+1\right) \times\left(2 N^{2}+\right.$ $2 N+1)$.

The computational complexities involved in obtaining the PLSI polynomial using the new way of form-preserving transformation are compared with the already existing method in the subsequent section.

\section{Computational Complexity}

This paper, discovered a new way of form-preserving transformation, which can be used for obtaining stable PLSI corresponding to the original unstable NSHP polynomial in a simple manner. Some theorems have been proposed in this context. In this section, the study of computational complexities involved with the new method and the existing method in the literature [10] is emphasized. In addition, comparison of the magnitude spectrum of original unstable filter, with the magnitude spectra of stable filters obtained, using the existing form-preserving transformation, proposed formpreserving transformation, and the reciprocity technique have been focused upon. It is obvious in this section that the proposed method to obtain PLSI polynomial is extremely simple, computationally efficient, and guarantees the design of stable NSHP filters. Obviously, in the literature the highest degree of most of the recursive filters considered in practice, goes up to 20 [18]. With this background in mind, the computational complexities have been computed in terms of total number of equations, total number of unknowns, and the dimension of $\bar{T}$ matrix for various degrees of polynomial up to 20 .

With the existing method, available in the literature, to obtain the PLSI polynomial, the form-preserving transformation, $A^{\prime}\left(z^{L}, z\right)$ where $L=4 N+1$ is applied. In the proposed method, it is shown that even with $L=$ $2 N+1$, stable PLSI can be obtained. From the figures shown in Table 1, it is evident that, there is an enormous reduction in computation in the proposed method and, therefore, it is less involved compared to existing method in the process of forming PLSI polynomial for the given NSHP polynomial.

\section{Summary and Conclusions}

This paper has discovered the design of stable 2D NSHP recursive digital filters using PLSI polynomials. The PLSI polynomial of the given 2D NSHP polynomial is derived using the new way of form-preserving transformation, and it was proved that the PLSI is always stable. It is known that obtaining PLSI directly from the 2D NSHP polynomial is laborious. The computational complexities involved in the discovered method have been compared with the existing method and it is demonstrated that the procedure is simple and computationally efficient. In this context, some theorems have been introduced. The strength of the paper is reinforced by adequate examples wherever 
needed. Some of the major conclusions arrived at are as follows.

(1) The proposed method always leads to an optimal LSI polynomial [25], and hence the stability of PLSI corresponding to the unstable 2D NSHP filter polynomial is always guaranteed.

(2) The stability test can be conducted at the end of optimization, and it is equivalent to a $1 \mathrm{D}$ test only.

(3) If the design reveals that the filter is unstable, taking PLSI of the denominator polynomial will make the transfer function stable, maintaining the magnitude spectrum at almost the same.

(4) Since the PLSI method works even for higher-order NSHP filters, direct design of stable NSHP filters is possible.

(5) If PLSI method is incorporated with the approximation method, then the design of NSHP filters will be guaranteed to be stable.

Theory of stabilization of 2D NSHP digital filters using the PLSI can be easily extended to the multidimensional $(\mathrm{mD})$ NSHP filters as well.

\section{References}

[1] I. F. Gonos, L. I. Virirakis, N. E. Mastorakis, and M. N. S. Swamy, "Evolutionary design of 2-dimensional recursive filters via the computer language GENETICA," IEEE Transactions on Circuits and Systems II, vol. 53, no. 4, pp. 254-258, 2006.

[2] V. M. Mladenov and N. E. Mastorakis, "Design of twodimensional recursive filters by using neural networks," IEEE Transactions on Neural Networks, vol. 12, no. 3, pp. 585-590, 2001.

[3] E. I. Jury, V. R. Kolavennu, and B. D. O. Anderson, "Stabilization of certain two-dimensional recursive digital filters," Proceedings of the IEEE, vol. 65, no. 6, pp. 887-892, 1977.

[4] J. W. Woods, I. Paul, and N. Sangal, "2-D direct form, recursive filter design with magnitude and phase specifications," in Proceedings of the IEEE International Conference on Acoustics, Speech, and Signal Processing (ICASSP'81), vol. 2, pp. 700-703, Atlanta, Ga, USA, March 1981.

[5] H. Chang and J. Aggarwal, "Design of two-dimensional semicausal recursive filters," IEEE Transactions on Circuits and Systems, vol. 25, no. 12, pp. 1051-1059, 1978.

[6] T. S. Huang, Ed., Two-Dimensional Digital Signal Processing I. Linear Filters, vol. 42 of Topics in Applied Physics, Springer, Berlin, Germany, 1981.

[7] M. N. S. Swamy, L. M. Roytman, and E. I. Plotkin, "Planar least squares inverse polynomials and practical-BIBO stabilization of $n$-dimensional linear shift-invariant filters," IEEE Transactions on Circuits and Systems, vol. 32, no. 12, pp. 1255-1260, 1985.

[8] N. Damera-Venkata, M. Venkataraman, M. S. Hrishikesh, and P. S. Reddy, "Stabilization of 2-D recursive digital filters by the DHT method," IEEE Transactions on Circuits and Systems II, vol. 46, no. 1, pp. 85-88, 1999.

[9] P. Delsarte, Y. Genin, and Y. Kamp, "Planar least squares inverse polynomials_part I: algebraic properties," IEEE
Transactions on Circuits and Systems, vol. 6, no. 1, pp. 59-66, 1979.

[10] N. Gangatharan and P. S. Reddy, "The PLSI method of stabilizing two-dimensional nonsymmetric half-plane recursive digital filters," EURASIP Journal on Applied Signal Processing, vol. 2003, no. 9, pp. 914-921, 2003.

[11] E. A. Robinson, Statistical Communication and Detection with Special Reference to Digital Data Processing of Radar and Seismic Signals, Hafner, New York, NY, USA, 1967.

[12] J. L. Shanks, S. Treitel, and J. H. Justice, "Stability and synthesis of two-dimensional recursive filters," IEEE Transactions on Audio and Electroacoustics, vol. 20, no. 2, pp. 115-128, 1972.

[13] Y. Genin and Y. Kamp, "Counterexample in the least-square inverse stabilization of 2D recursive filters," Electronics Letters, vol. 11, pp. 130-131, 1975.

[14] Y. Genin and Y. Kamp, "Comments on "on the stability of the least mean-square inverse process in two-dimensional digital filters"', IEEE Transactions on Acoustics, Speech and Signal Processing, vol. 25, no. 1, pp. 92-93, 1977.

[15] L. F. Chaparro, "Asymmetric half-plane planar least-squares inverses," in Proceedings of the IEEE International Conference on Acoustics, Speech, and Signal Processing (ICASSP '82), vol. 7, pp. 2072-2075, Paris, France, May 1982.

[16] L. F. Chaparro and R. Bournas, "Sufficient conditions for minimum phase two-dimensional PLSI's," IEEE Transactions on Acoustics, Speech and Signal Processing, vol. 32, no. 4, pp. 916-918, 1984.

[17] A. H. Kayran and R. King, "Comments on least-square inverse polynomials and a counterexample for Jury's conjecture," Electronics Letters, vol. 16, no. 21, pp. 795-796, 1980.

[18] J. S. Lim, Two-Dimensional Signal and Image Processing, Prentice-Hall, Englewood Cliffs, NJ, USA, 1989.

[19] M. P. Ekstrom and J. W. Woods, "Two-dimensional spectral factorization with applications in recursive digital filtering," IEEE Transactions on Acoustics, Speech and Signal Processing, vol. 24, no. 2, pp. 115-128, 1976.

[20] P. S. Reddy, D. R. R. Reddy, and M. N. S. Swamy, "Proof of a modified form of Shanks' conjecture on the stability of 2-D planar least square inverse polynomials and its implications," IEEE transactions on circuits and systems, vol. 31, no. 12, pp. 1009-1015, 1984.

[21] P. K. Rajan and H. C. Reddy, "Formulation of 2-D normal equations using 2-D to 1-D form preserving transformations," IEEE Transactions on Acoustics, Speech and Signal Processing, vol. 36, no. 3, pp. 414-417, 1988.

[22] J. L. Shanks, "The design of stable two-dimensional recursive filters," in Proceedings of the UMR-Mervin J. Kelly Communications Conference, pp. 15-2-1-15-2-5, Rolla, Mo, USA, October 1970.

[23] J. L. Shanks, "Two planar digital filtering algorithms," in Proceedings of the 5th Princeton Conference on Information Sciences and Systems, pp. 48-53, Princeton, NJ, USA, March 1971.

[24] P. Delsarte, Y. Genin, and Y. Kamp, "Comments on 'proof of a modified form of Shanks' conjecture on the stability of 2-D planar least square inverse polynomials and its implications," IEEE Transactions on Circuits and Systems, vol. 32, no. 9, p. 966, 1985.

[25] P. K. Rajan and H. C. Reddy, "A note on LSI polynomials and comments on a 'proof of a modified form of Shanks' conjecture," IEEE Transactions on Circuits and Systems, vol. 32, no. 9, pp. 966-968, 1985. 
[26] B. T. O'Connor and T. S. Huang, "Stability of general twodimensional recursive digital filters," IEEE Transactions on Acoustics, Speech and Signal Processing, vol. 26, no. 6, pp. 550560, 1978.

[27] K. R. Santhi, N. Gangatharan, and M. Ponnavaikko, "A novel approach for testing stability of 1-D recursive digital filters based on lagrange multipliers," American Journal of Applied Sciences, vol. 5, no. 5, pp. 490-495, 2008. 